\title{
MASTER
}

\section{SEMI-ANNUAL STATUS REVIEW}

NOVEMBER 1971

* NOZZLE, EXTENSION, PRESSURE VESSEL

L. A. SHURLEY 


\section{DISCLAIMER}

This report was prepared as an account of work sponsored by an agency of the United States Government. Neither the United States Government nor any agency Thereof, nor any of their employees, makes any warranty, express or implied, or assumes any legal liability or responsibility for the accuracy, completeness, or usefulness of any information, apparatus, product, or process disclosed, or represents that its use would not infringe privately owned rights. Reference herein to any specific commercial product, process, or service by trade name, trademark, manufacturer, or otherwise does not necessarily constitute or imply its endorsement, recommendation, or favoring by the United States Government or any agency thereof. The views and opinions of authors expressed herein do not necessarily state or reflect those of the United States Government or any agency thereof. 


\section{DISCLAIMER}

Portions of this document may be illegible in electronic image products. Images are produced from the best available original document. 


\title{
SEMI-ANNUAL STATUS REVIEW
}

\author{
NOVEMBER 1971
}

PRESSURE VESSEL AND CLOSURE

L. A. SHURLEY 


\section{PRESSURE VESSEL SIGNIFICANT EVENTS \\ E/CDSR TO NOVEMBER 1971}

- COMPLETED AND PUBLISHED PRESSURE VESSEL MID-CYLINDER THERMAL DESIGN STUDY REPORT

- COMPLETED STEADY-STATE EOL THERMAL ANALYSIS OF PV CYLINDER WITH FLOW PERTURBATORS

- COMPLETED STEADY-STATE EOL THERMAL ANALYSIS OF FORWARD CLOSURE JOINT WITH 7075-T73 CLOSURE

- COMPLETED STEADY-STATE EOL THERMAL ANALYSIS OF 7075-T73 CLOSURE

- COMPLETED 7075-T73 CLOSURE LAYOUT AND DETERMINED WEIGHT SAVINGS RESULTING FROM MATERIAL CHANGE

- UPDATED PVC DESIGN LAYOUT REFLECTING CLOSURE MATERIAL CHANGE

- COMPLETED REVIEW AND APPROVAL OF TWO ISSUES OF PVC C-002 SPECIFICATION

- UPDATED PVC FAILURE-MODE ANALYSIS 
PRESSURE VESSEL

PLANNED ACTIVITY

NOVEMBER 1971 TO MAY 1972

- UPDATE PVC DESIGN LAYOUT REFLECTING CHANGES REQUIRED FROM ANALYSES AND INTERFACE DEFINITION

- CONDUCT STRESS ANALYSIS OF FORWARD JOINT

1

- CONDUCT STRESS ANALYSIS OF 7075-T73 CLOSURE

- PERFORM PROBABILISTIC RELIABILITY ANALYSIS OF SELECTED CRITICAL FAILURE MECHANISMS

- ISSUE DRAFT OF PVC DESIGN REPORT

- CONTINUE COORDINATION WITH WANL TO RESOLVE FORWARD REACTOR INTERFACE DEFINITION 
MID-CYLINDER DESIGN STUDY

STEADY-STATE EOL THERMAL ANALYSIS

- CYLINDER WITH FLOW PERTURBATORS

- FWD CLOSURE JOINT (7075-T73)

- 7075-T73 CLOSURE SHELL

STEADY-STATE EOL STRESS ANALYSIS

- FWD JOINT (7075-T73)

- 7075-T73 CLOSURE SHELL

PREPARE DRAFT OF PVC DESIGN REPORT

RELIABILITY

- UPDATE PVC FMA

- CALCULATE PRELIMINARY

RELIABILITY OF SELECTED

CRITICAL FAILURE MECHANISMS

7075-T73 LAYOUT AND WEIGHT SAVINGS

UPDATE PVC DESIGN LAYOUT

SUPPORT FOR C-002 SPEC. PREPARATION

PVC/NSS INTERFACE COORDINATION

WITH WANL

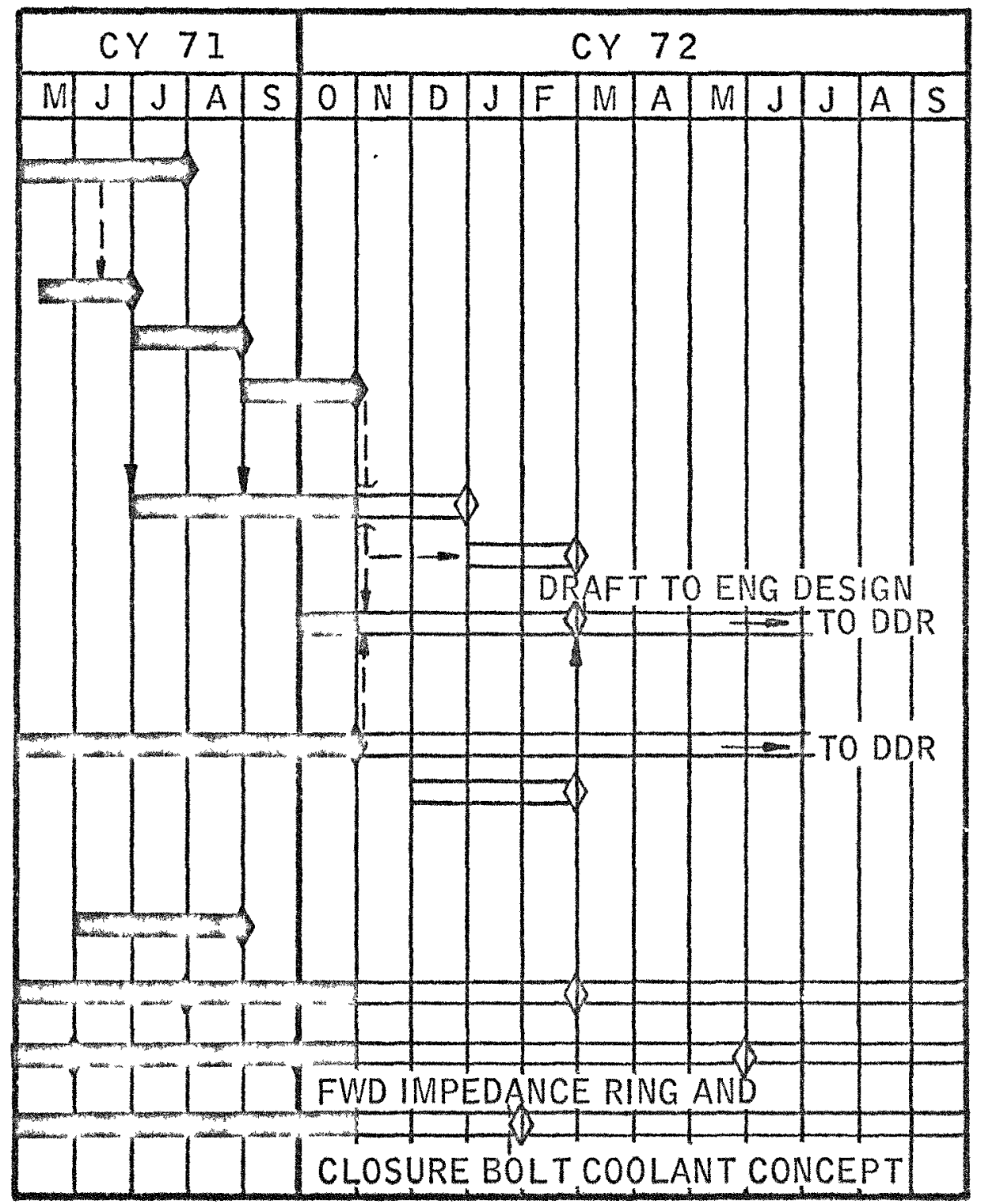

TiY AEROJET nUCLEAR SYSTEMS COMPAHY 


\section{PRESSURE VESSEL \\ SELECTED TECHNICAL TOPICS}

- CLOSURE MATERIAL CHANGE 6061 TO 7075 AA

- FWD IMPEDANCE RING LOCATION AND BOLT COOLANT

- MID-CYLINDER OVERHEATING RESOLUTION 
NON-WELDED INSTRUMENTATION PORT ADAPTER CONCEPT

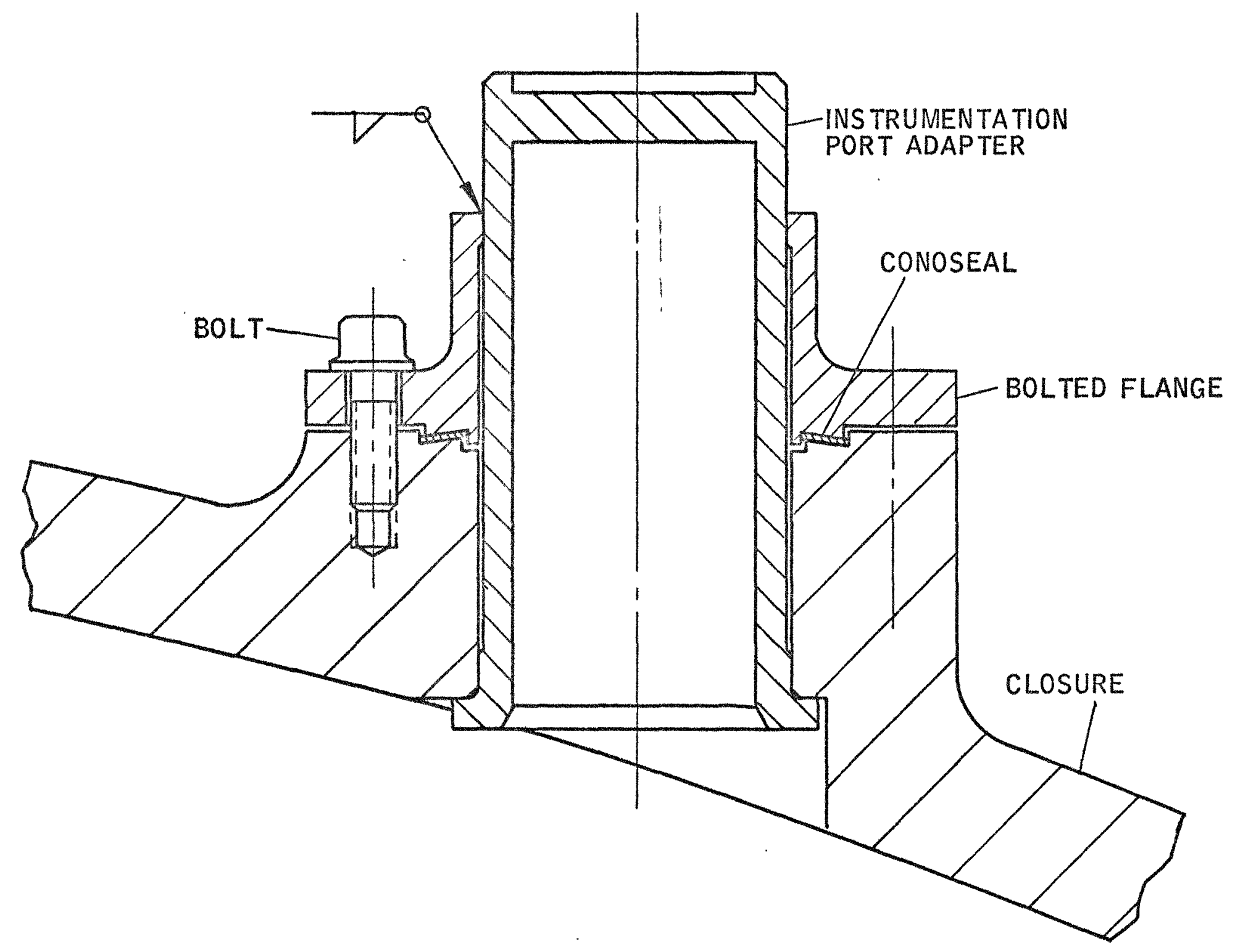

010 aerojet nuclear systems company 
PRESSURE VESSEL CYLINDER MID-WALL TEMPERATURE PROFILE

STEADY-STATE EOL CONDITIONS

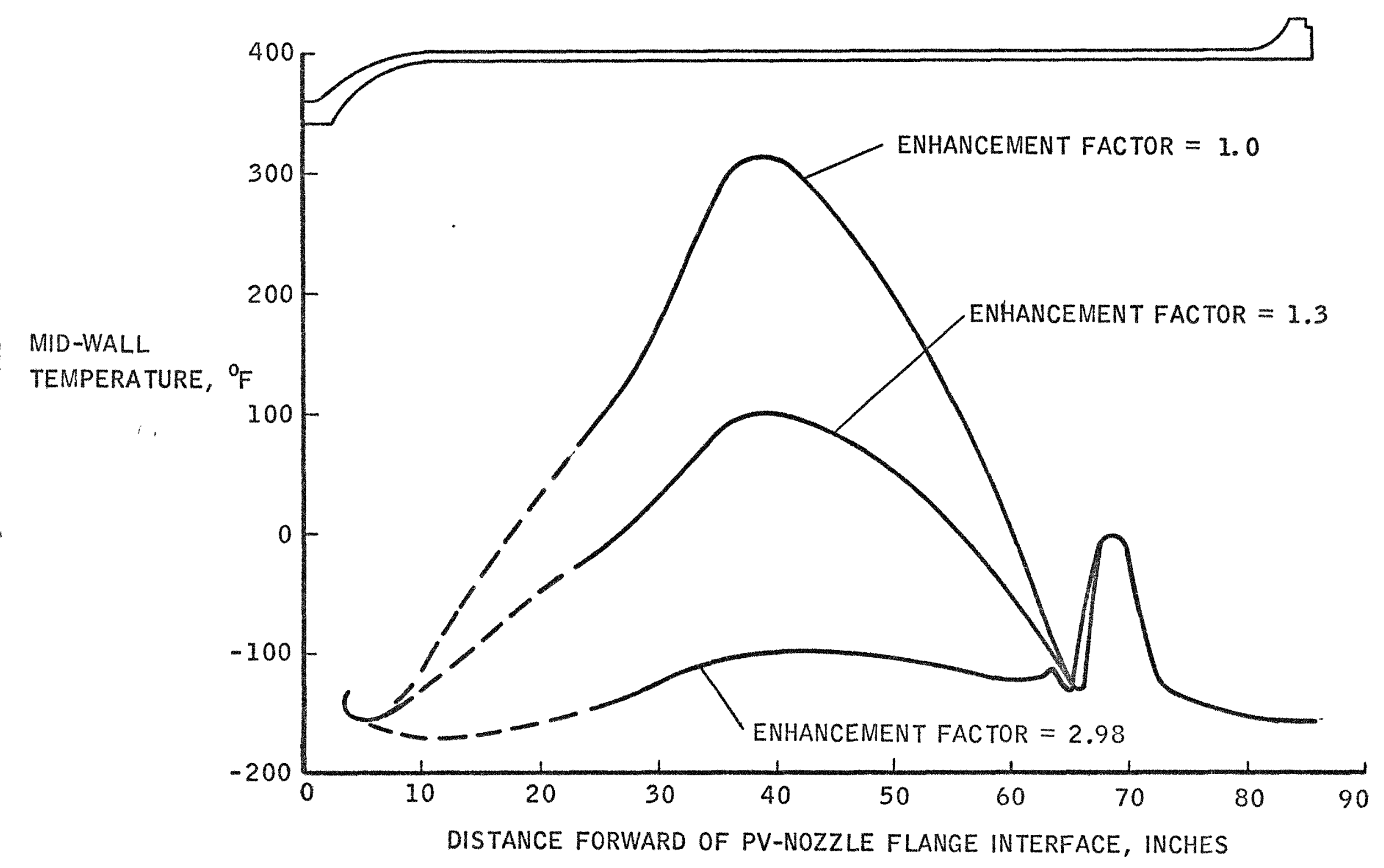

97. AEROJET nUCLEAR SYSTEMS COMPANY 
CLOSURE BOLT COOLANT CONCEPT

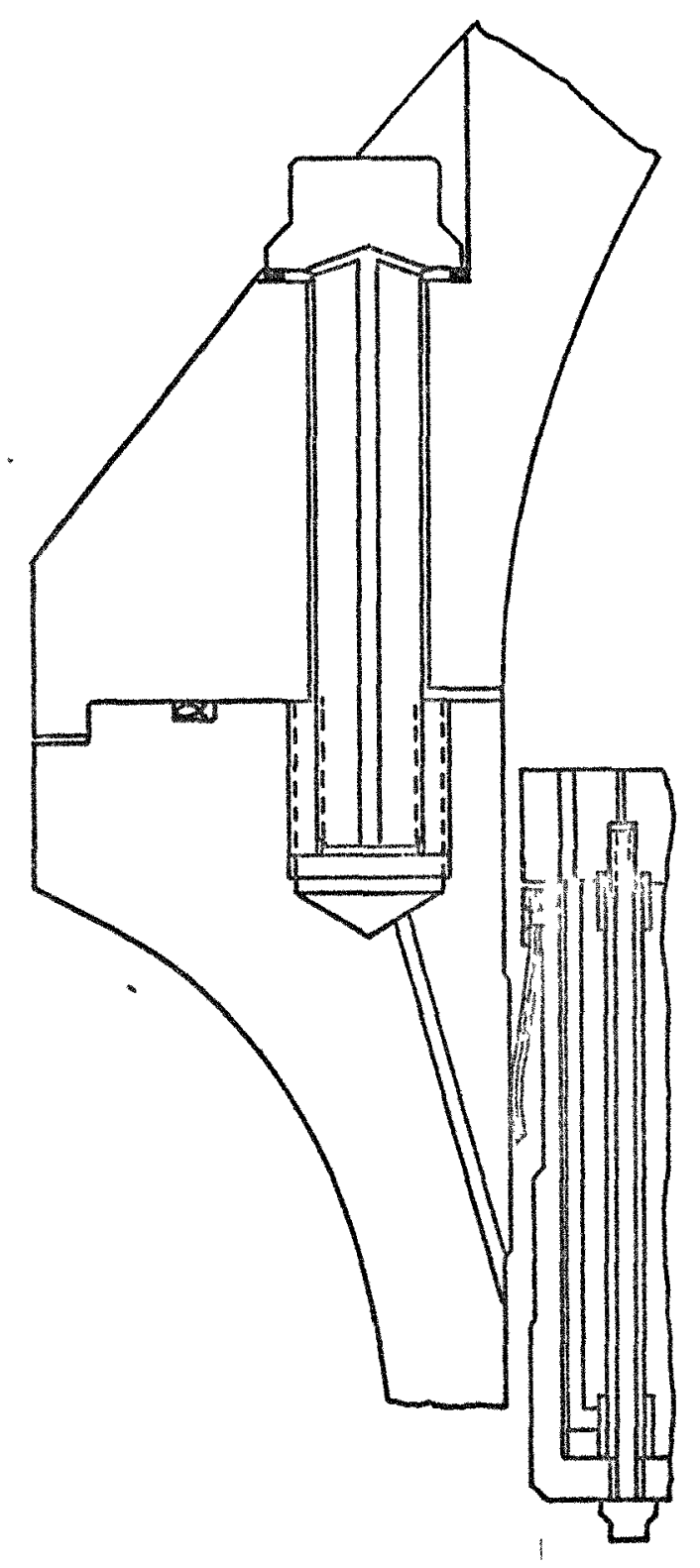




\begin{tabular}{|c|c|c|c|c|}
\hline & \multicolumn{4}{|c|}{ PRELIMINARY PROBABILISTIC ANALYSIS } \\
\hline & \multicolumn{2}{|c|}{ MAY 71 VALUES } & \multicolumn{2}{|c|}{ NOV 71 VALUES } \\
\hline & ALLOCATED & ASSESSED & ALLOCATED & ASSESSED \\
\hline $\begin{array}{l}\text { PVC FAILS TO CONTAIN } \\
\text { PROPELLANT } \\
\text { O LEAKAGE AT NOZZLE TO PV } \\
\text { JOINT } \\
\text { O LEAKAGE AT PV CLOSURE } \\
\text { JOINT }\end{array}$ & $\begin{array}{l}\cdot 96 \\
.9686 \\
.9664\end{array}$ & $\begin{array}{l}\text { TBD } \\
\text { TBD }\end{array}$ & $\begin{array}{l}.987 \\
.9963 \\
.9963\end{array}$ & $\begin{array}{l}\text { TBD } \\
\text { TBD }\end{array}$ \\
\hline $\begin{array}{l}\text { PVC FAILS TO TRANSMIT ENGINE } \\
\text { LOADS AND PROVIDE STRUCTURAL } \\
\text { SUPPORT FOR INTERFACING COM- } \\
\text { PONENTS } \\
\text { O STRUCTURAL FAILURE OF PV } \\
\text { WALL } \\
\text { O FAILURE OF PV TO CLOSURE } \\
\text { JOINT } \\
\text { O SHEAR LIP FAILURE OF PV } \\
\text { CLOSURE } \\
\text { OFAILURE OF PV CLOSURE } \\
\text { BETWEEN BOLT HOLES }\end{array}$ & $\begin{array}{l}\cdot{ }^{9} 5^{88} \\
.9664 \\
\cdot{ }^{6} 6^{64} \\
.97^{4} \\
.97^{4}\end{array}$ & $\begin{array}{c}.{ }^{9} 7^{5 * *} \\
.{ }^{9} 3^{8} \\
>{ }^{9}{ }_{10} \\
>{ }^{9} 10\end{array}$ & $\begin{array}{l}.{ }^{9} 10^{0} \\
.9 \\
{ }^{1} 1^{5} \\
{ }^{9}{ }^{1} 1^{5} \\
.{ }^{9} 11^{5} \\
.{ }^{9} 11^{5}\end{array}$ & $\begin{array}{l}\text { TBD } \\
.{ }^{9} 11^{5 *} \\
\text { TBD } \\
\text { TBD }\end{array}$ \\
\hline
\end{tabular}

* an enhancement factor on heat transfer coefficient of 1.60 is required TO PRODUCE THIS VALUE

* THIS VALUE WAS BASED ON TEMPERATURES THAT SUBSEQUENT ANALYSIS PROVED INACCURATE 


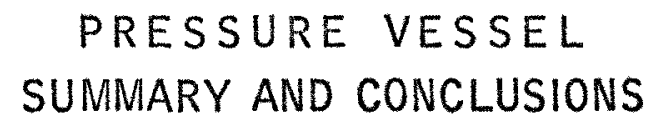

TECHNICAL RESULTS

- WEIGHT SAVINGS OF 95 LBS RESULTED FROM CLOSURE MATERIAL CHANGE

- WITH A COOLING ENHANCEMENT FACTOR OF 1.6 THE CYLINDER TEMPERATURE AT MID-CORE IS $10^{\circ} \mathrm{F}$, WHICH PROVIDES A M.S. OF +.11 AND A RELIABILITY OF.${ }^{9} 115$.

- MAXIMUM CLOSURE FLANGE TEMPERATURE IS $-142^{\circ}$

- MAXIMUM CLOSURE BOLT (UNCOOLFD) TEMPERATURE IS $-99^{\circ} \mathrm{F}$ PROJECT STATUS

- ALL WORK STATEMENTS ON SCHEDULE 